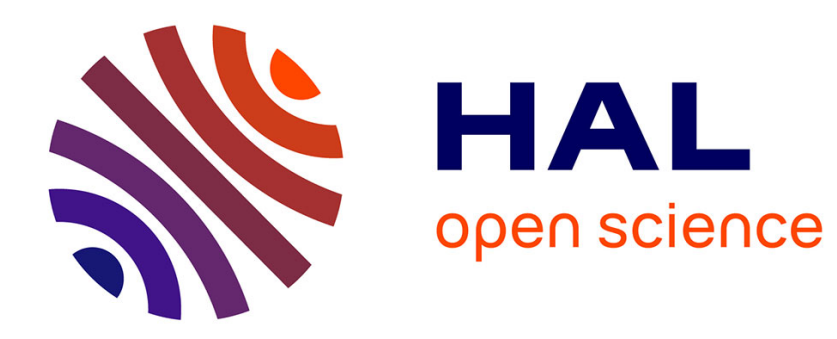

\title{
Doser rapidement l'austénite résiduelle avec précision
}

F. Convert, B. Miege

\section{To cite this version:}

F. Convert, B. Miege. Doser rapidement l'austénite résiduelle avec précision. Journal de Physique IV Proceedings, 1996, 06 (C4), pp.C4-863-C4-878. 10.1051/jp4:1996483 . jpa-00254366

\section{HAL Id: jpa-00254366 https://hal.science/jpa-00254366}

Submitted on 1 Jan 1996

HAL is a multi-disciplinary open access archive for the deposit and dissemination of scientific research documents, whether they are published or not. The documents may come from teaching and research institutions in France or abroad, or from public or private research centers.
L'archive ouverte pluridisciplinaire HAL, est destinée au dépôt et à la diffusion de documents scientifiques de niveau recherche, publiés ou non, émanant des établissements d'enseignement et de recherche français ou étrangers, des laboratoires publics ou privés. 


\title{
Doser rapidement l'austénite résiduelle avec précision
}

\author{
F. Convert et B. Miege \\ Service Analyses Physiques, CETIM, 52 avenue Félix Louat, 60304 Senlis cedex, France
}

\begin{abstract}
Résumé : Le contrôle de l'austénite résiduelle dans les aciers est un besoin industriel. Le dosage de cette austénite par diffractométrie $X$ en dispersion d'énergie est avant tout une méthode d'analyse rapide. Grâce à la modélisation du spectre de diffraction ${ }^{1}$, nous avons créé un outil précis, peu sensible aux phénomènes de texture et pas du tout affecté par la présence de carbures en teneur importante. On peut traiter des spectres de diffraction enregistrés avec un très faible taux de comptage, ce qui autorise des analyses sur de petites zones, moyennant une augmentation raisonnable des temps d'acquisition. Dans le domaine d'utilisation de 0 à $100 \%$ de phase, sa précision est équivalente à la diffractométrie conventionnelle, voire supérieure pour les très faibles teneurs. Nous avons ainsi mis au point un outil parfaitement adapté au contrôle industriel.
\end{abstract}

\begin{abstract}
Retained austenite control in steels is an industrial necessity. First of all, retained austenite measurements by EDPD (Energy Dispersive Powder Diffraction) is a very fast method of analysis. Due to our rebuilding of the diffraction pattern ${ }^{1}$, we perfected an accurate tool not very sensitive to prefered orientation and which is not troubled by the presence of high carbides amounts. Low counting rate diffraction datas can be analysed, so measurements on small specimen areas can easily be carried out, provided that adequate counting time is selected. This technique is available in the range $0 \%-100 \%$ of austenite content with the same accuracy as conventional X-Ray diffractometry and even better for low concentration. With such features, we performed a technique which seems to be perfectly adapted to industrial testings.
\end{abstract}

\section{INTRODUCTION}

L'austénite résidı̉elle est une phase que l'on trouve en plus ou moins grande quantité dans les aciers ou les fontes, après traitement thermique. Elle doit être souvent éliminée en partie, ou au contraíre acceptée en grande quantité, selon le traitement effectué et les propriétés recherchées pour la tenue en service des pièces. Elle doit donc être contrôlée. Pour cela, on dispose d'un certain nombre de techniques, telles que :

- la mesure de l'aimantation à saturation qui nécessite un étalonnage et ne permet le dosage qu'en volume, sur des échantillons de dimensions standards.

- la spectrométrie Mössbauer, précise mais inadaptée à la pratique industrielle.

- Le contrôle micrographique purement qualitatif, mais que certains tentent de rendre quantitatif par l'emploi d'images types ou par l'utilisation de l'analyse d'image, pour des aciers dont la structure permet la visualisation de l'austénite.

- La diffractométrie $X$ conventionnelle qui permet un dosage précis relativement localisé en surface, mais qui s'avère en pratique souvent long et onéreux, surtout lorsque l'on ne veut analyser qu'une zone très réduite de quelques $\mathrm{mm}^{2}$. En cas de texture du matériau, il faut faire

\footnotetext{
${ }^{1}$ Rapport interne CETIM-Senlis, Le Dosage Rapide de l'Austénite Résiduelle, fait par F.Convert et B.Miège, le 30 mars 1995
} 
tourner l'échantillon pendant la mesure, et donc utiliser un support particulier, mais le temps d'analyse est alors beaucoup plus long.

- La diffractométrie $\mathrm{X}$ à angle fixe couplée à un détecteur dispersif en énergie, autorise des enregistrements très courts, ce qui est très intéressant sur le plan du contrôle industriel. Mais elle n'est pas vraiment employée jusqu'à présent, bien qu'elle soit connue depuis que les premiers détecteurs à semi-conducteur $\mathrm{Si}$-Li soient apparus sur le marché, il y a plus de 20 ans.

Les laboratoires de contrôle utilisent surtout la diffractométrie $\mathrm{X}$ conventionnelle pour sa précision. Parmi les applications les plus courantes, on peut citer :

- Les pièces de métrologie dimensionnelle (calibres,...), où le taux d'austénite peut être élevé. On n'en accepte généralement que quelques pour-cents pour éviter les déformations dues’à sa transformation dans le temps.

- Les roulements, qui doivent contenir très peu d'austénite, car elle se transforme par frottement en cours d'utilisation. Les dosages sur de très petites zones (fond de gorge de roulement par exemple) peuvent durer de 10 à $20 \mathrm{~h}$.

- Les engrenages cémentés, dans lesquels la teneur en austénite est souvent élevée (souvent 20 à 40\%). La teneur doit être optimisée afin obtenir la meilleure tenue en service.

- D'une manière générale, le contrôle de l'austénite résiduelle est utilisé pour la mise au point de gammes de traitement thermique. Pour les traiteurs à façon, la technique de diffractométrie $\mathrm{X}$ conventionnelle est longue et coûteuse, et ils utilisent parfois le contrôle micrographique. L'apparition de normes pourrait à terme contraindre ces traiteurs à réaliser plus de contrôles. Comme tous les industriels, ils auront donc besoin d'une technique rapide et fiable, applicable à toutes les nuances d'aciers, quelles que soient leurs structures.

\section{LA DIFFRACTOMETRIE PAR DISPERSION D'ENERGIE}

Cette méthode a déjà été présentée par de nombreux auteurs [1]..[6], et nous renvoyons le lecteur à ces articles de référence.

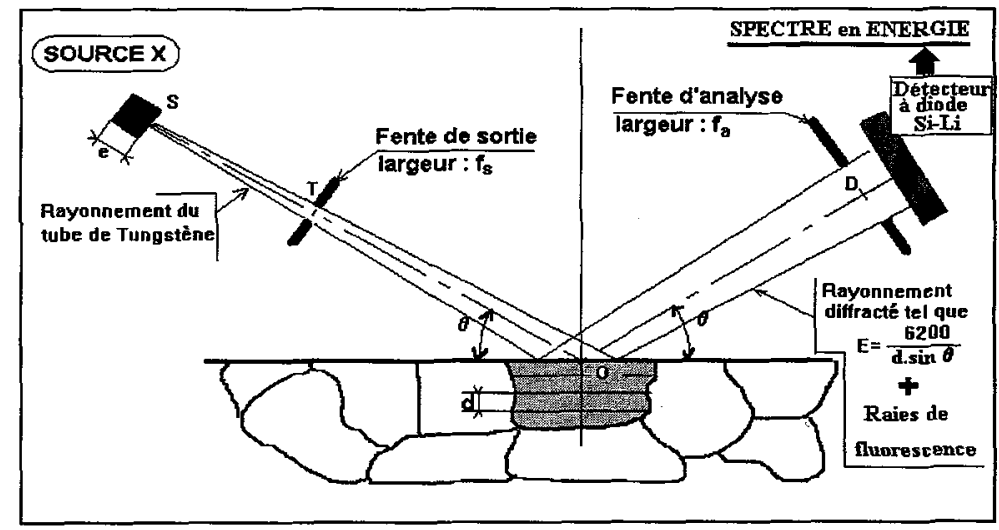

Figure 1 : Schéma du montage expérimental. $\mathrm{SO}=250 \mathrm{~mm} \mathrm{OD}=140 \mathrm{~mm} \mathrm{ST}=86 \mathrm{~mm}$. HT : $50 \mathrm{kV}$ A schematic of the experimental setting. 
Le montage expérimental est représenté figure 1. La source de rayonnement est un tube de tungstène dont le spectre en énergie est présenté figure 2. Ce rayonnement irradie l'échantillon dont la surface fait un angle $\theta$ avec le faisceau incident.

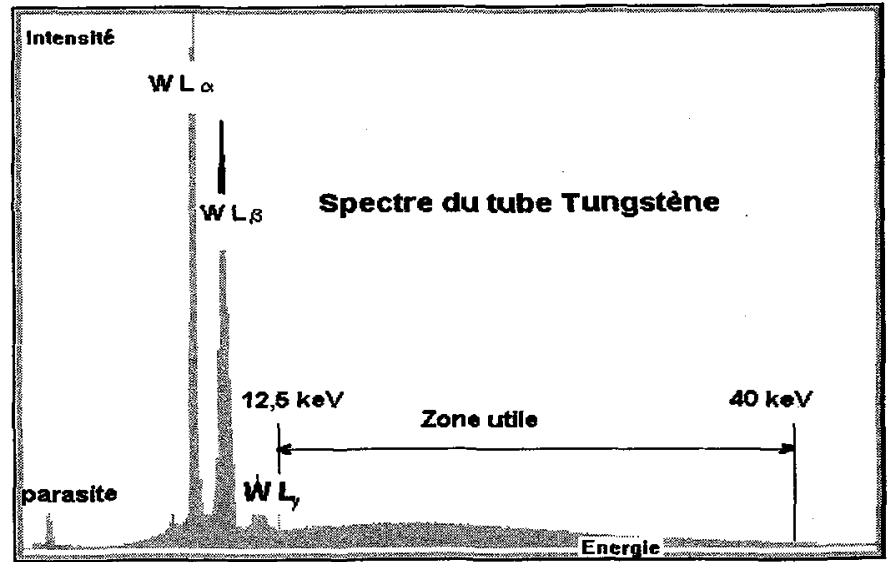

Figure 2 : Spectre en énergie du rayonnement $X$ provenant d'une anode de tungstène. $\mathrm{X}$-Ray energy pattern from a tungsten target tube.

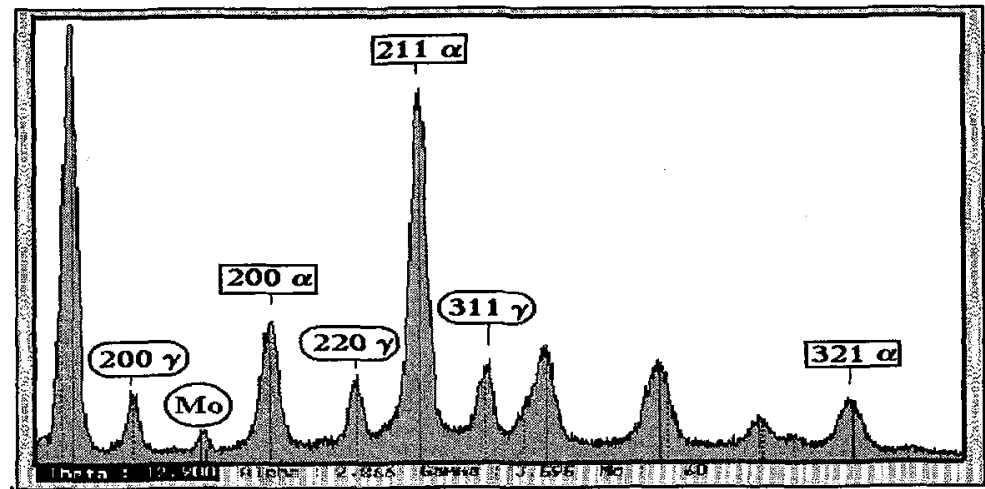

Figure 3 : Spectre de diffraction en dispersion d'énergie d'un acier avec $22 \%$ d'austénite résiduelle $\left(\theta=13^{\circ}\right)$. $X$-Ray energy diffraction pattern of steel containing $22 \%$ retained austenite $\left(\theta=13^{\circ}\right)$.

Les photons du rayonnement incident ne seront diffractés par une famille de plans (hkl), ayant une distance interatomique $d_{h k l}$, que s'ils possèdent une énergie $E_{h k l}$ donnée par la relation de Bragg :

$$
\mathrm{E}_{h k 1}=\frac{6200}{d_{h k l} \cdot \sin \theta}
$$


Ces photons seront diffractés avec un angle $\theta$ symétrique par rapport à la normale au plan diffractant. En mettant un détecteur dans la direction faisant un angle $\theta$ avec la surface de l'échantillon, on recueille le rayonnement provenant des plans parallèles à la surface.

En utilisant un détecteur à semi-conducteur, par exemple une diode $\mathrm{Si}-\mathrm{Li}$, on peut trier les photons en énergie, c'est-à-dire compter le nombre de photons $\mathrm{X}$ qui arrivent dans le détecteur pour chaque énergie $E_{h k l}$. On obtient ainsi un spectre de diffraction en énergie tel que celui de la figure 3. Il est constitué d'un ensemble de raies dont les positions en énergie correspondent chacune à une valeur de $\mathrm{d}_{\mathrm{hkl}}$ (relation 1 ). Ce spectre ne concerne que la zone énergétique qui correspond à la partie continue du spectre de la source de tungstène, c'est-à-dire $12-40 \mathrm{keV}$. A l'angle $\theta=13^{\circ}$ auquel a été pratiqué l'enregistrement, 21 raies de l'acier sont présentes, mais seulement 6 sont bien séparées des autres.

Le rayonnement incident excite aussi les atomes des divers éléments présẹnts aans la matrice. Des rayonnements de fluorescence sont donc émis par ces atomes, et recueillis par le détecteur. Ces raies viennent se superposer aux raies de diffraction. En pratique, dans les aciers, les seuls éléments susceptibles de donner un rayonnement de fluorescence dans le domaine supérieur à $12 \mathrm{keV}$ sont le molybdène et le nobium.

Plus l'angle d'analyse est élevé, et plus l'énergie des photons diffractés par une famille de plans donnée est faible. Dans ces conditions, en augmentant l'angle du montage, on décale certaines raies dans le domaine $\mathrm{E}<12 \mathrm{keV}$, tandis que d'autres apparaissent dans le domaine utile. Comme il en apparaît plus qu'il n'en disparaît, le nombre de raies du spectre augmente avec l'angle tandis que l'intensité globale diminue.

\section{DOSAGE DE L'AUSTENITE RESIDUELLE PAR DIFFRACTOMETRIE X}

\subsection{Intensité nette des raies de diffraction}

\subsubsection{En diffraction conventionnelle,}

on utilise un rayonnement monochromatique, et le spectre est enregistré en mesurant l'intensité en fonction de l'angle. Dans ce cas, on connaît [7] la formule donnant l'intensité nette intégrée, $I(\lambda)$.

$$
\mathrm{I}(\lambda)=\mathrm{h} \cdot \mathrm{S}(\lambda) \cdot \frac{\lambda^{3}}{V_{c}^{2}} \cdot m \cdot F_{h k l}^{2} \cdot \frac{1+\cos ^{2} 2 \theta}{\sin ^{2} \theta \cdot \cos \theta} \cdot \mathrm{D}(\lambda, \theta) \cdot \frac{1}{\mu(\lambda)} \cdot \Delta v
$$

A noter que l'intensité n'est connue qu'à un facteur multiplicatif près, $\mathbf{h}$. L'intensité nette intégrée $I(\lambda)$ de la raie de diffraction, centrée sur l'angle $\theta$ est inversement proportionnelle :

- au coefficient d'absorption $\mu$, qui varie avec $\lambda$.

- au carré du volume de maille, $\mathrm{V}_{\mathrm{c}}{ }^{2}$

proportionnelle à :

- l'intensité de la source $S(\lambda)$,

- au facteur de multiplicité m,

- au carré du facteur de structure $\mathrm{F}^{2} h k l$,

- au facteur de température ou de Debye, $D(\lambda, \theta)$,

- à une fonction de $\lambda$

- à une fonction de l'angle $\theta$,

- au volume de la phase $\Delta v$ à l'origine de la raie diffractante. 
L'intensité intégrée est proportionnelle au volume $\Delta \mathrm{V}_{\varphi}$ de la phase $\varphi$ qui diffracte, et à une fonction $\mathrm{K}\left(\varphi, \lambda, \theta_{\mathrm{hkl}}\right)$. La formule donnant l'intensité peut donc se résumer à :

$$
\mathrm{I}(\varphi, h k l)=\mathrm{h} \cdot \mathrm{K}\left(\varphi, \lambda, \theta_{h k l}\right) \cdot \Delta V_{\varphi}
$$

Le coefficient $K$, qui fait intervenir les paramètres énumérés ci-dessus, se calcule numériquement [8] à partir des données de la littérature [9]. Pour un rayonnement donné, ces coefficients peuvent être calculés une fois pour toutes, pour chaque famille de plan (hkl), car l'intensité de la source $S(\lambda)$ qui est une constante, est prise en compte dans la constante $h$.

\subsubsection{En diffractométrie par dispersion d'énergie,}

les fonctions intervenant dans la relation (2) doivent être modifiées en tenant compte de la relation fondamentale reliant la longueur d'onde à l'énergie $\lambda=\frac{12400}{E}$ ( $\lambda$ est exprimé en Angströms et $\mathrm{E}$ en électron-volts). Appelons W(E) le spectre en énergie de la source X. On trouvera un exemple de l'allure de cette fonction figure 4. La relation entre longueur d'onde et énergie implique que $\mathrm{S}(\lambda)=E^{2} \mathrm{~W}(E)$. Après avoir effectué toutes les transformations nécessaires dans l'expression (2), l'intensité s'écrit sous la forme d'un produit de fonctions :

$$
\mathrm{I}(\varphi, \mathrm{hkl})=\operatorname{Cste} \cdot \mathrm{F}(\theta) \cdot \mathrm{G}\left(\mathrm{E}_{\mathrm{hkl}}\right) \cdot \mathrm{H}\left(\varphi, \mathrm{d}_{\mathrm{hkl}}\right) \cdot \mathrm{L}(\varphi) \cdot \Delta \mathrm{V}_{\varphi}
$$

$\mathrm{F}:$ ne dépend que de l'angle d'analyse, c'est donc une constante.

$\mathrm{G}$ : est une fonction de l'énergie, qui inclus notamment le coefficient d'absorption du Fer et le spectre de la source.

$H$ : est fonction des $d_{h k l}$ de la phase $\varphi$. Elle inclus le coefficient de température et les facteurs de multiplicité des raies.

$\mathrm{L}:$ ne dépend que de la phase. Elle inclus le facteur de structure et le carré du volume de maille.

On peut écrire comme en diffractométrie conventionnelle : $(\varphi, h k l)=\mathrm{h} . \mathrm{K}\left(\varphi, E_{h k}, d_{h k l}\right) \cdot \Delta V_{\varphi}$

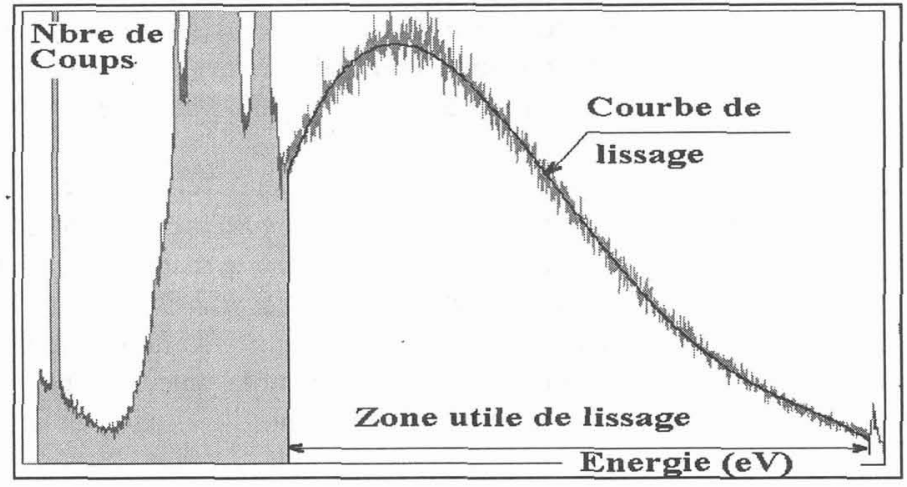

Figure 4 : Agrandissement du spectre en énergie de la source représenté figure 2. Magnification of the pattern of figure 2. 
Contrairement à la diffractométrie conventionnelle, le spectre en énergie $W(E)$, qui intervient dans la fonction $G$, n'est pas uniforme, et on doit le prendre en compte dans le calcul du coefficient $\mathrm{K}$. La détermination de ce coefficient $\mathrm{K}$ se révèle donc plus complexe qu'en diffractométrie conventionnelle, car il dépend étroitement de la répartition énergétique du rayonnement incident ainsi que de l'angle auquel on travaille. C'est sans doute un des points les plus gênants de la méthode, qui a dû limiter son développement.

On notera aussi l'intérêt de travailler avec une anode constituée d'un élément lourd comme le tungstène, car l'intensité du spectre continu est proportionnelle au numéro atomique $\mathrm{Z}$. L'intensité des spectres, proportionnelle à cette intensité par l'intermédiaire du coefficient $\mathrm{K}$, sera donc plus importante avec ce type d'anode.

\subsection{Application au dosage de l'austénite}

Que ce soit en diffractométrie conventionnelle ou par dispersion d'énergie, l'intensité de la raie s'exprime par une relation de la forme :

$$
\mathrm{I}(\varphi, h k l)=\mathrm{h} \cdot \mathrm{K}(\varphi, h k l) \cdot \Delta \bar{V}_{\varphi}
$$

Pour un montage donné, les coefficients $K$ peuvent être déterminés numériquement, tandis que $h$ et $\Delta V_{\varphi}$ sont inconnus. Appelons $C$ la teneur en carbures supposée connue. Soit $I_{\gamma}$ l'intensité d'une raie de la phase gamma dont la teneur est $\gamma_{R}$, et $\mathrm{I}_{\alpha}$ l'intensité d'une raie de la phase alpha dont la teneur est $1-\gamma_{\mathrm{R}}-\mathrm{C}$. On montre alors facilement que la teneur en phase gamma est donné par la relation:

$$
\gamma_{R}=(1-\mathrm{C}) \frac{\mathrm{I}_{\gamma}, \mathrm{K}(\alpha, h k l)}{\mathrm{I}_{\gamma}, \mathrm{K}(\alpha, h k l)+\mathrm{I}_{\alpha} \cdot \mathrm{K}(\gamma, h k l)}
$$

Il suffit de disposer des intensités intégrées d'une seule raie alpha et d'une seule raie gamma pour déterminer la quantité de chacune des phases. Il est malheureusement très courant que les matériaux analysés soient plus ou moins texturés. Dans ce cas, les coefficients théoriques $\mathrm{K}(\varphi$ ,hkl ) ne sont plus corrects. Il s'ensuit une erreur sur la détermination de $\gamma_{R}$ que l'on peut diminuer en utilisant plusieurs raies de chaque phase. Dans l'exemple de la figure 3 , on voit que seulement 3 raies gamma et 3 raies alpha sont bien séparées des raies voisines. On dispose ainsi de 6 relations pour seulement 2 inconnues. Pour calculer ces 2 valeurs, on peut utiliser des méthodes de pondération comme la méthode de BEU [10]. Mais il est tout aussi simple d'employer une méthode des moindres carrés.

L'utilisation de plus de raies serait intéressante pour pondérer l'effet de texture qui est très fréquent. Malheureusement, comme les raies se chevauchent fortement, le calcul de l'intensité nette de chaque raie ne peut se faire qu'en utilisant une méthode mathématique de séparation des raies. Le çalcul le plus général qui permet de traiter ce problème consiste à faire une reconstitution mathématique du spectre complet, en ajustant numériquement certains paramètres inconnus. Ce traitement fournit alors directement la teneur en phase recherchée, puisqu'elle fait partie des paramètres à ajuster numériquement. 


\section{PRINCIPES DE LA MODELISATION DU SPECTRE DE DIFFRACTION EN DISPERSION D'ENERGIE}

Appelons I(E) l'intensité du spectre de diffraction correspondant à l'énergie E. C'est la somme des contributions de chacune des phases et du fond à cette énergie (plus éventuellement la contribution de la fluorescence de certains éléments). Les calculs théoriques précédents ne s'appliquent qu'aux intensités nettes intégrées. Pour déterminer la contribution de chaque raie, il est nécessaire de modéliser la forme des raies de diffraction du spectre.

\subsection{Modélisation d'une raie de diffraction :}

En première approximation, on peut décrire la répartition en énergie par une fonction symétrique, centrée sur la valeur $E_{h k l}$ donnée par la relation (1). Les fonctions de Gauss ou de Cauchy correspondent par exemple à cette définition. Ce sont des fonctions $F(x)$ paires telles que $\mathrm{F}(0)=1$ et $\mathrm{F}(\mathrm{x}) \rightarrow 0$ lorsque $|\mathrm{x}| \rightarrow \infty . \mathrm{x}=\frac{E-E_{h k l}}{L}$ est une variable centrée réduite. On appelle $I_{\max }$ l'intensité maximale de cette raie, et $L$ la largeur à mi-hauteur. L'intensité de la raie de diffraction correspondant à la valeur $d_{h k l}$ peut se modéliser par $I_{h k l}(E)=I_{\max } \cdot F(x)$.

Par ailleurs, on a vu que l'intensité nette intégrée de cette raie est $\mathrm{I}_{\text {nette }}(\mathrm{hkl})=\mathrm{h} \cdot \mathrm{K}(\varphi, h k l) \cdot \Delta V_{\varphi}$. Par définition, elle est égale à l'aire de la fonction $\mathrm{F}(\mathrm{x})$, soit $\mathrm{I}_{\text {nette }}=\mathrm{I}_{\max } \cdot \int_{-\infty}^{\infty} \mathrm{F}(x) \cdot \mathrm{d} E$. Comme $\mathrm{d} x=\frac{\mathrm{d} E}{L}$ et que l'intégrale est finie, il est facile de montrer que $I_{\max } \propto \frac{I_{\text {nette }}}{L}$. L'intensité de la raie de la phase $\varphi$ peut donc se modéliser par l'expression :

$$
\mathrm{I}_{\varphi}(E, h k l)=\mathrm{u} \cdot \Delta V_{\varphi} \cdot \frac{\mathrm{K}(\varphi, h k l)}{L} \cdot \mathrm{F}\left(\frac{E-E_{h k l}}{L}\right)
$$

dans laquelle $u$ est une constante inconnue.

\subsection{Modélisation de la largeur de la raie}

La largeur de la raie de diffraction est le résultat du cumul des élargissements provoqués par chaque élément du montage. Il existe 3 élargissements principaux qui sont dus :

- à la dispersion du détecteur

- à la dispersion due aux fentes du montage et à la taille de la source.

- au matériau (taille des domaines cohérents et dispersion sur les $\alpha_{h k l}$ ).

\subsection{1 élargissement dû au détecteur :}

La raie de diffraction reconstituée par un détecteur à semi-conducteur $\mathrm{Si}$ - $\mathrm{Li}$ est une gaussienne dont la largeur à mi-hauteur $\mathrm{L}_{\text {det }}$ est de la forme :

$$
\mathrm{L}_{\operatorname{det}}^{2}(E)=\mathrm{A}+\mathrm{B} \cdot E
$$




\subsection{1 élargissement dî au montage :}

Les fentes d'analyse et de sortie, ainsi que la taille de la source, provoquent chacune une dispersion angulaire $\Delta \theta$ de l'angle d'analyse $\theta$. Cela entraine une dispersion en énergie $\Delta \mathrm{E}$ que l'on peut relier à la dispersion angulaire par la relation $\Delta E=E \cdot \operatorname{cotg} \theta . \Delta \theta$, obtenue en différenciant la relation (1). La répartition de l'intensité dans chaque intervalle $\Delta \mathrm{E}$ est une répartition uniforme (en forme de créneau), sauf pour la divergence axiale. Pour simplifier la suite des calculs, nous avons admis que la loi de répartition de l'intensité introduite par chacune de ces divergences suit une loi de Gauss, dont l'aire est égale à celle du créneau. On montre alors que la largeur à mi-hauteur $\mathrm{L}_{\mathrm{g}}$ de cette gaussienne équivalente doit être telle que $\mathrm{L}_{g}=\sqrt{\frac{2}{3}} \cdot \Delta E \approx 0,82 \cdot \Delta E$. Cette hypothèse permet alors de dire que la répartition de l'intensité due à l'élargissement total du montage est une gaussienne, dont le carré de la largeur, $\mathrm{l}^{2} \mathrm{~m}$, est égal à la somme des carrés des largeurs de chacun des élargissements dus au montage.

Si on appelle $\Delta \theta_{\mathrm{i}}$ la dispersion angulaire provoquée par chaque élément du montage, on en déduit tous calculs faits :

$$
1_{m}^{2}=\frac{2}{3} \cdot\left[\operatorname{cotg}^{2} \theta \cdot \sum_{i} \Delta \theta_{i}^{2}\right] E^{2}
$$

Les valeurs des $\Delta \theta_{\mathrm{i}}$ se calculent facilement à partir de considérations géométriques. On peut écrire d'une manière plus concise : $1_{m}=\mathrm{u} . \mathrm{f}($ montage). $E$. La pseudo-variable montage regroupe les variables largeurs des fentes, taille de la source et de la zone d'analyse, distance source-échantillon et échantillon-détecteur, position de la fente d'analyse, et bien entendu, angle de Bragg du montage.

\subsection{3 élargissement dû au matériau :}

4.2.3.1 la taille des domaines diffractants diminuant, la largeur des raies augmente, d'après la formule de Scherrer [7]. A partir de cette formule, adaptée au cas de la diffractométrie en dispersion d'énergie, on montre que cet élargissement en énergie est un terme assez petit qui ne dépend pas de l'énergie $E$. Il est de la forme $\frac{k}{\sin \theta}$.

4.2.3.2 La dispersion sur le paramètre de maille, $\frac{\Delta a}{a}$, peut devenir le principal élargissement des raies du spectre. Comme les phases alpha et gamma ont des mailles cubiques, on montre facilement que cet élargissement $1_{\mathrm{a}}$ est de la forme $1_{a}=\mathrm{p} . E$, où $\mathrm{p}$ est une constante qui ne dépend que du matériau.

\subsubsection{Elargissement total :}

En ajoutant les carrés des largeurs de tous ces élargissements, la largeur des pics de diffraction est donnée par : 


$$
\begin{aligned}
& \mathrm{L}^{2}(E)=\mathrm{L}_{\mathrm{det}}^{2}+\mathrm{l}_{\mathrm{m}}^{2}+\mathrm{l}_{\mathrm{a}}^{2}=\mathrm{A}+\mathrm{B} \cdot E+\mathrm{Q}^{2} \cdot E^{2} \\
& \operatorname{avec} \mathrm{Q}^{2}=\mathrm{u}^{2} \cdot \mathrm{f}^{2}(\text { montage })+\mathrm{p}^{2} \quad\left(\mathrm{u}^{2}=\frac{2}{3}\right) \\
& \text { et } \mathrm{A}^{*}=\mathrm{A}+\frac{\mathrm{k}^{2}}{\sin ^{2} \theta}
\end{aligned}
$$

\subsection{Modélisation du spectre}

Les considérations précédentes sur la largeur nous ont conduit à décrire les raies de diffraction par une gaussienne, dont la largeur à mi-hauteur est donnée par la relation (5). L'intensité d'une raie de la phase $\varphi$ s'écrit donc :

$$
\mathrm{I}_{\varphi}(E, h k l)=\mathrm{u} \cdot \Delta V_{\varphi} \cdot \mathrm{F}_{\varphi}(E, h k l) \text { avec } \mathrm{F}_{\varphi}(E, h k l)=\frac{\mathrm{K}(\varphi, h k l)}{\mathrm{L}_{\varphi}\left(E_{h k l}\right)} \cdot \exp \left[-\ln 2 \cdot \frac{\left(E-E_{h k l}\right)^{2}}{\mathrm{~L}_{\varphi}^{2}\left(E_{h k l}\right)}\right]
$$

L'intensité correspondant à la contribution de la phase $\varphi$ dans le spectre est la somme de ces intensités, c'est-à-dire :

$$
\mathrm{I}_{\varphi}(E)=\sum_{h k l} \mathrm{I}_{\varphi}(E, h k l)=\mathrm{u} . \Delta V_{\varphi} \cdot \sum_{h k l} \mathrm{~F}_{\varphi}(E, h k l)
$$

L'intensité totale, correspondant à l'énergie $\mathrm{E}$, est la somme des contributions du fond Fond(E), et des contributions $\mathrm{I}_{\alpha}(\mathrm{E})$ et $\mathrm{I}_{\gamma}(\mathrm{E})$ des phases alpha et gamma, ce que l'on peut écrire:

$$
\mathrm{I}(E)=\operatorname{Fond}(E)+\mathrm{u} . \sum_{\varphi=\alpha}^{e=\alpha}\left\{\Delta V_{\varphi} \cdot \sum_{h k l} \mathrm{~F}_{\varphi}(E, h k l)\right\}
$$

De plus, dans les spectres d'analyse, on constate presque toujours la présence de raies de fluorescence du molybdène, qui est un élément souvent présent dans les aciers.

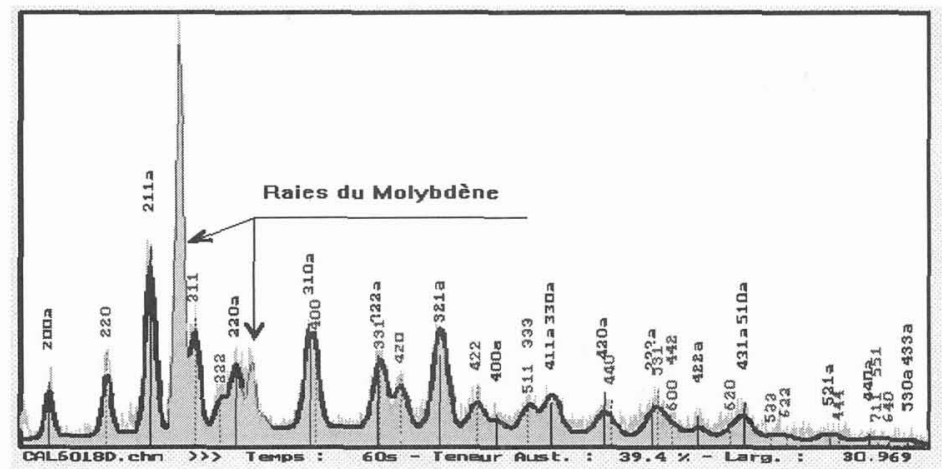

Figure 5 : Spectre de diffraction d'un acier contenant environ $40 \%$ d'austénite résiduelle. $\theta=18,5^{\circ}$ $\mathrm{X}$-Ray energy dispersive diffraction pattern of steel containing $40 \%$ retained austenite. 
Pour le calcul, on peut soit exclure cette zone de l'analyse, soit au contraire inclure ces raies dans la modélisation. C'est cette dernière solution qui a été retenue. Il faut donc ajouter un terme supplémentaire à cette expression. Ce terme se modélise facilement par des sommes de gaussiennes dont les positions et les largeurs sont connues, mais dont les intensités doivent être déterminées dans le calcul du spectre.

La modélisation du fond peut se faire par un polynôme. Dans ce cas, les coefficients du polynôme doivent être ajustés avec les autres paramètres inconnus.

Le résultat de l'ajustement numérique permet d'accéder aux teneurs en phases alpha et gamma, sachant que $\Delta \mathrm{V}_{\alpha}+\Delta \mathrm{V}_{\gamma}=1-\mathrm{C}$, où $\mathrm{C}$ représente la teneur en carbures.

La figure 5 donne un exemple de spectre typique obtenu pour un angle $\theta=18,5^{\circ}$. En trait épais, le résultat de la modélisation. Les indices (hkl) des plans qui diffractent sont reportés en face de chacune des raies. La lettre $a$ qui suit certains indices indique qu'il s'agit d'une raie de la phase alpha, dont la position est marquée en trait plein. On peut remarquer la présence de raies supplémentaires qui sont les raies de fluorescence du molybdène.

Lorsque l'on constate un excellent accord entre l'expérience et la modélisation, comme c'est le cas sur la figure 5, cela indique que le matériau analysé n'est pas texturé.

\section{VERIFICATION EXPERIMENTALE}

Cette vérification expérimentale a porté essentiellement sur la validité de la modélisation de la largeur des raies, et la validité du calcul du modèle appliqué à des valeurs très diverses de l'angle $\theta$ du montage.

\subsection{Modélisation de la largeur}

Pour vérifier la modélisation de la largeur, nous avons procédé à l'enregistrement du spectre d'un acier ferritique pour diverses conditions de fentes et d'angles de Bragg :

- fente d'analyse comprise entre 0,2 et $1 \mathrm{~mm}$

- fente de sortie comprise entre 0,2 et $2 \mathrm{~mm}$

- angle de Bragg variant de $5^{\circ}$ à $18,5^{\circ}$

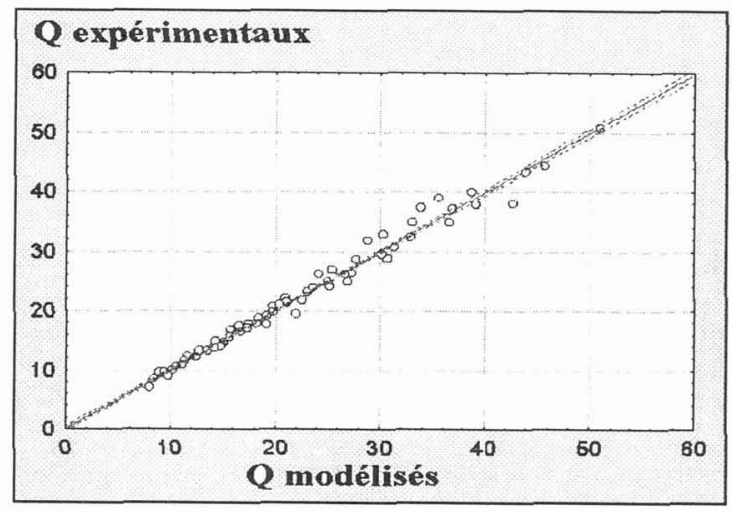

Figure 6 : Corrélation entre les valeurs de $Q$ expérimentales et modélisées. Correlation between experimental and modeled $Q$ values. 
Nous avons relevé à chaque fois la valeur du facteur $Q$ qui intervient dans la relation (5), en négligeant le terme d'élargissement dû à la taille des domaines diffractants.

La fonction $\mathrm{f}$ (montage) a été calculée théoriquement à partir des conditions géométriques du montage intervenant dans la relation (4). Nous avons ensuite ajusté par régression les valeurs de $\boldsymbol{u}$ et $\boldsymbol{p}$ qui interviennent dans l'expression modélisée de $Q$

Le résultat de ce calcul a donné une valeur de $u$ voisine de 0,7 , ce qui est en très bon accord avec la théorie, car on peut montrer que la détermination de u est entachée d'une grande erreur. La comparaison entre valeurs théoriques et déterminées par le logiciel est représentée par le graphe de la figure 6.

La bonne corrélation obtenue entre la valeur de $Q$ modélisée, et celle déterminée par notre logiciel confirme la validité de la modélisation de la largeur introduite dans notre calcul.

\subsection{Analyse en fonction de l'angle de Bragg}

La seconde vérification de la modélisation que nous avons réalisée, a consisté à faire des analyses sur différentes sortes d'échantillons, à des angles très différents. En effet, si la modélisation est correcte, le résultat ne doit pas dépendre de l'angle de Bragg utilisé, sauf si le matériau est texturé.

Tableau 1 : Dosage de l'austénite en fonction de $\theta$, pour un acier traité $\left(\mathrm{N}^{\circ} 1\right)$ et un étalon $\mathrm{NBS}^{\circ}\left(\mathrm{N}^{\circ} 2\right)$. Austenite measured values versus $\theta$ for hardened steel $\left(\mathrm{N}^{\circ} 1\right)$ and $\mathrm{NBS}$ standard $\left(\mathrm{N}^{\circ} 2\right)$

\begin{tabular}{|c|c|c|c|c|c|c|c|c|c|c|c|c|}
\hline Angle $\theta\left({ }^{\circ}\right)$ & 13 & 13,5 & 14 & 14,5 & 15 & 15,5 & 16 & 16,5 & 17 & 17,5 & 18 & 18,5 \\
\hline $\mathrm{N}^{\circ} 1(19 \%)$ & 22,3 & 22,5 & 21,2 & 22,2 & 22,5 & 22,2 & 21,3 & 21,3 & 21,4 & 21,5 & 21,9 & 21,9 \\
\hline $\mathrm{N}^{\circ} 2(4,0 \%)$ & 5,4 & 5,7 & 4,9 & 4,9 & 5,2 & 4,7 & 4,3 & 4,8 & 4,6 & 4,5 & 4,9 & 4,3 \\
\hline
\end{tabular}

Tableau 2 : Dosage de l'austénite en fonction de $\theta$, pour 2 étalons NBS. Austenite measured values versus $\theta$ for 2 NBS standards.

\begin{tabular}{|c|c|c|c|c|}
\hline $\begin{array}{c}\text { Angle } \theta \text { de } \\
\text { Bragg }\left({ }^{\circ}\right)\end{array}$ & \multicolumn{2}{|c|}{ Nombre de raies hkl de la phase } & \multicolumn{2}{c|}{ Teneur en phase austénitique (\%) } \\
\cline { 2 - 5 } & ALPHA & GAMMA & NBS1 (2,9\%) & NBS2 (29,5\%) \\
\hline 7,25 & 2 & 3 & 2,7 & 30,0 \\
\hline 7,8 & 3 & 3 & 3,1 & 30,3 \\
\hline 9,5 & 4 & 5 & 3,5 & 29,7 \\
\hline 12 & 7 & 8 & 3,5 & 30,1 \\
\hline 14 & 10 & 11 & 3,6 & 29,7 \\
\hline 15,5 & 12 & 14 & 2,7 & 28,3 \\
\hline 17 & 14 & 16 & 2,4 & 30,0 \\
\hline 18,5 & 18 & 20 & 2,9 & 29,6 \\
\hline
\end{tabular}

Pour faire cette vérification, nous avons procédé à des enregistrements de spectres à différents angles de Bragg, pour divers matériaux tels que :

- un étalon dont la teneur moyenne en phase gamma annoncée par le fournisseur est de $4,0 \%\left(\mathrm{~N}^{\circ} 2\right.$, tab. 1). Cette moyenne a été déterminée à partir des résultats obtenus par diffractométrie $X$, analyse d'image et mesures magnétiques. 
- 2 échantillons (tab.2) contenant respectivement $2.33 \%$ et $33.89 \%$ d'austénite, d'après le fournisseur. Les teneurs en austénite mesurées par nos soins, en diffractométrie conventionnelle, sont indiquées entre parenthèses. Comme le précédent, ces 2 échantillons sont un mélange de 2 aciers obtenu par frittage. Le premier est un acier austénitique contenant $20 \%$ de nickel, le second est un acier ferritique, sans nickel. La teneur en austénite a été obtenue par analyse d'image au microscope, et cette teneur a été corrélée à l'intensité du pic de nickel obtenu par fluorescence $\mathrm{X}$. Les teneurs données par le fournisseur sont donc le résultat de cette analyse par fluorescence $X$ sur le nickel de nos témoins.

- un acier sur lequel nous nous étions assuré de l'absence de texture, par analyse diffractométrique conventionnelle de l'austénite, et qui avait conduit à une valeur de $19 \%$. (tab. 1)

Les résultats des tableaux 1 et 2 montrent une très grande stabilité de l'analyse en fonction de l'angle $\theta$. En particulier, on constate sur le tableau 2 que le nombre total de raies passe de 5 à 38 , sans que cela affecte notablement le résultat de l'analyse. Les résultats sont en accord avec les dosages effectués par diffractométrie $\mathrm{X}$ conventionnelle, pour tous les échantillons. L'écart avec la valeur annoncée $(33,89 \%)$ pour l'échantillon NBS2 est bien plus grand que ce que la reproductibilité des mesures impliquerait. Par des dosages suivant le diamètre de l'échantillon, on a par contre mis en évidence une forte hétérogénéité de structure (variation de $26 \%$ à $32 \%$ d'un bord à l'autre de l'échantillon). Par ailleurs, la méthode d'analyse de l'austénite par analyse d'image et fluorescence $X$, est sans doute moins précise que ce que le fournisseur annonce $( \pm 0,5 \%)$. Tout ceci peut expliquer cet écart.

\subsection{Influence de certains paramètres sur les résultats d'analyse}

\subsubsection{Influence du temps de comptage}

Les résultats d'analyse seront d'autant plus reproductibles que la fluctuation des comptages sera faible, c'est-à-dire que l'intensité du spectre sera forte. Pour cela, il faut disposer d'une grande surface irradiée, mais ce n'est pas toujours le cas. Il faut alors augmenter les temps de comptage. N'ayant pas encore fait de calcul de reproductibilité de la mesure, nous avons procédé à quelques essais pour obtenir un ordre de grandeur des précisions obtenues, en fonction des temps de comptage. Des analyses sur les échantillons NBS frittés, ont été réalisées sur une surface de $5 \times 12 \mathrm{~mm}^{2}$, avec des temps de comptages variables. Les résultats sont reportés dans le tableau 3. Les teneurs mesurées en diffractométrie conventionnelle sont reportées entre parenthèses.

Tableau 3 : Influence du temps de comptage sur l'analyse à $\theta=18,5^{\circ}$.

Counting time effect upon measurement results.

\begin{tabular}{|c|c|c|c|c|c|c|}
\hline \multicolumn{2}{|c|}{ temps de comptage (s) } & 30 & 60 & 120 & 300 & 600 \\
\hline Teneur en & NBS1 (2,9\%) & 1,6 & 2,6 & 2,9 & 2,8 & 2,9 \\
\hline$\gamma_{\mathrm{R}}(\%)$ & NBS2 (29,5\%) & 29,3 & 28,9 & 28,8 & 28,3 & 28,9 \\
\hline
\end{tabular}

On constate dans le tableau 3 que le résultat de l'analyse n'est plus modifié au bout d'une minute. Une minute de comptage est typiquement ce que l'on peut utiliser dans les cas courants. Si l'on ajoute à cela 40 s de calcul pour modéliser le spectre, on conçoit la rapidité avec laquelle on peut maintenant réaliser un dosage. 
Tableau 4 : Influence du temps de comptage sur l'analyse d'une bague de roulement (impact : $1 \times 2 \mathrm{~mm}^{2}, \theta=15^{\circ}$ ) Counting time effect upon measurement in ball-race bearing (measured area : $1 \times 2 \mathrm{~mm}^{2}, \theta=15^{\circ}$ )

\begin{tabular}{|c|c|c|c|c|c|}
\hline comptage (s) & 100 & 400 & 800 & 1600 & 2400 \\
\hline$\gamma_{\mathrm{R}}(\%)$ & 31,3 & 35,5 & 36,4 & 35,5 & 35,9 \\
\hline
\end{tabular}

L'exemple du tableau 4 concerne l'analyse de l'austénite au fond d'une gorge de bague de roulement, pour laquelle l'impact du faisceau était de $2 \mathrm{~mm}^{2}$. On voit qu'à $400 \mathrm{~s}$ ont obtient déjà une valeur identique à celles obtenues avec des temps de comptage plus élevés. Le dosage de l'austénite dans la même zone, réalisé en diffractométrie $\mathrm{X}$ conventionnelle, à donné une valeur de $33 \%$, mais $15 \mathrm{~h}$ d'enregistrement ont été nécesaires pour obtenir un spectre exploitable.

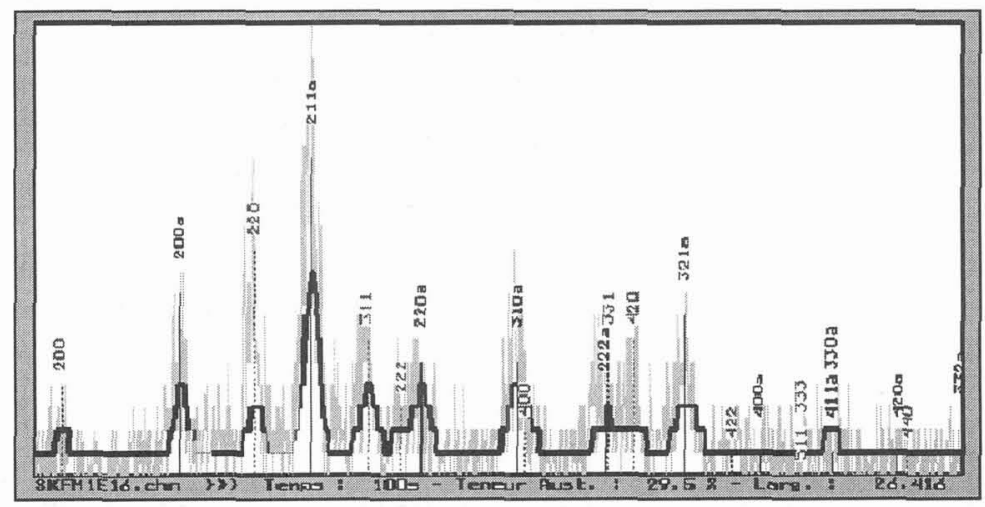

Figure 7 : Spectre correspondant à l'enregistrement de 100s (voir tableau 4).

Diffraction pattern corresponding to the 100 s recording (see table 4).

Le spectre de la figure 7 correspond à l'enregistrement de 100 s. Il met en évidence l'efficacité de la modélisation du spectre, car les intensités ne dépassent pas quelques dizaines de coups au sommet de la raie la plus intense, alors que l'écart à la valeur en austénite n'excède pas $4 \%$, d'après le tableau 4.

\subsubsection{Influence de la quantité de phase sur la précision des dosages}

Il est intéressant de pouvoir préciser le domaine de validité de ce type d'analyse. Après modélisation du spectre, on détermine la teneur en austénite à partir d'une formule équivalente à la relation (3). L'étude de cette relation montre alors que les phases alpha et gamma jouent un rôle équivalent dans la détermination de la teneur en austénite ou ferrite. On doit donc obtenir la même sensibilité de la méthode pour doser la ferrite en petite quantité.

Pour le vérifier, nous avons procédé à la mesure de la ferrite sur 2 alliages d'Inconel, dans lequel une mesure de la ferrite par technique magnétique décelait une teneur de $0,2 \%$ et $2 \%$. La mesure par diffractométrie $\mathrm{X}$ nous a donné respectivement des teneurs de $0-0,1 \%$ et $2-3 \%$. Pour ce qui concerne le seuil de détection, il n'est lié qu'à la dispersion statistique des comptages qui conditionne la précision statistique de la mesure. On peut donc toujours 
diminuer ce seuil en augmentant l'intensité du spectre par l'accroissement du temps de comptage. Le domaine de validité de la méthode s'étend donc de $0 \%$ à $100 \%$.

\subsubsection{Influence d'une erreur de positionnement de l'échantillon}

Un problème qui peut aussi se poser dans ce type d'analyse est une erreur de positionnement de l'échantillon par rapport à la référence. Il en résulte une modification de l'angle de Bragg que le calcul détermine par ajustement numérique. Pour vérifier l'inocuité d'une telle erreur de positionnement, nous avons décalé volontairement le porte-échantillon de $\pm 2 \mathrm{~mm}$ par rapport à notre référence. Comme l'impact se déplace alors d'une valeur 3 fois plus grande pour un angle $\theta$ de $18,5^{\circ}$, nous avons à chaque fois déplacé l'échantillon, afin d'analyser toujours la même zone.

Tableau 5 : Influence d'un défaut de positionnement sur le résultat de l'analyse. (impact : 1x10 $\mathrm{mm}^{2}$ ).

Sample missetting effect upon measurements results (spot : $1 \times 10 \mathrm{~mm}^{2}$ ).

\begin{tabular}{|c|c|c|c|c|c|c|c|}
\hline décalage $(\mathrm{mm})$ & -2 & -1 & $-0,5$ & 0 & $+0,5$ & +1 & +2 \\
\hline angle $\theta\left(^{\circ}\right)$ & 19,27 & 18,89 & 18,69 & 18,48 & 18,28 & 18,09 & 17,73 \\
\hline$\gamma_{\mathrm{R}}(\%)$ & 28,3 & 29,8 & 27,2 & 27,6 & 27,5 & 27,6 & 30,2 \\
\hline
\end{tabular}

Les résultats présentés dans le tableau 5 montrent que le décalage n'a pas d'influence sur le résultat, car les fluctuations mesurées correspondent à l'incertitude de la méthode, pour cette teneur en austénite.

\subsubsection{Matériaux contenant des carbures}

L'analyse de l'austénite dans des aciers contenant des carbures en assez grande quantité, est souvent impossible en diffractométrie conventionnelle, si la teneur en austénite est faible, car les raies des carbures viennent alors se superposer aux raies de l'austénite, pour les domaines habituels de l'analyse.

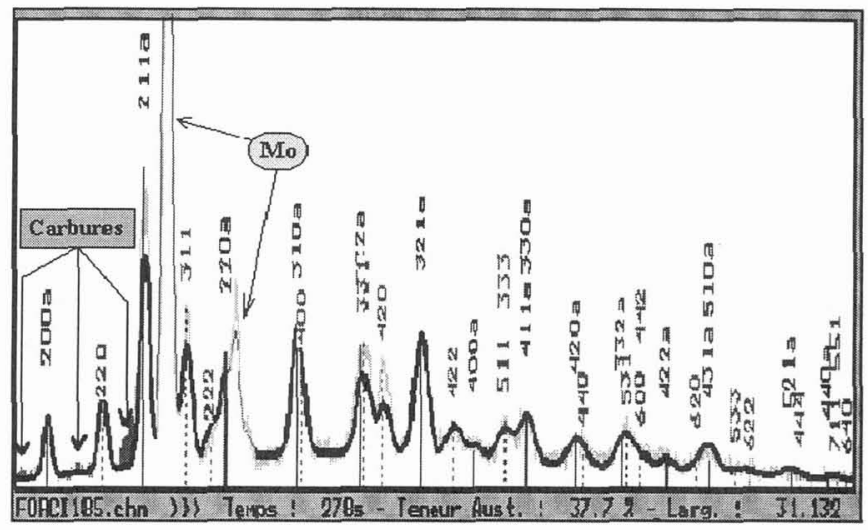

Figure 8 : Spectre d'une fonte au chrome contenant environ $30 \%$ de carbures. $\left(\theta=18,5^{\circ}\right)$

Diffraction pattern of high chromium cast iron containing $30 \%$ carbides. $\left(\theta=18,5^{\circ}\right)$ 
En diffractométrie à en angle fixe, les raies des carbures sont rejetées vers les basses énergies, si on utilise des angles de diffraction relativement élevés. Seules quelques raies peu intenses subsistent alors dans le spectre d'analyse, comme on peut le voir sur le spectre de la figure 8 qui correspond à celui d'une fonte au chrome, contenant environ $30 \%$ de carbures (estimation par micrographie). On constate que les raies de carbures ne sont pas venues perturber la modélisation du spectre.

\subsubsection{Matériaux texturés}

La texture est sans doute le problème le plus difficile à résoudre en diffractométrie conventionnelle. Un des moyens de résoudre le problème est de faire tourner l'échantillon pendant la mesure, à l'aide d'un support adapté.

En diffractométrie à angle fixe, on sait qu'il est possible de réaliser une analyse qui porte sur un très grand nombre de raies, dès lors que l'on travaille aux grands angles. La modélisation qui prend en compte l'ensemble des raies, produit alors un effet de pondération important sur les effets de texture. De plus, aux grands angles, les raies $(110)_{\alpha}$ et $(111)_{\gamma}$, les plus sensibles à l'extinction primaire [11][12], sont absentes de la zone énergétique utilisée. Enfin, le spectre contient alors des raies ayant un facteur de multiplicité élevé, moins sensibles à la texture [12][13].

Afin de vérifier que le fait de travailler aux grands angles pouvait permettre de limiter l'erreur introduite par la présence de texture, nous avons effectué une série d'analyses sur un acier austéno-ferritique, dont la teneur en austénite est classiquement de 50-55\%. Cet acier présente en effet une forte texture sur certaines raies, comme le résultat d'une analyse à un angle $\theta$ de $18,5^{\circ} 1^{\prime}$ a mis en évidence (voir figure 9). Les résultats sont rassemblés dans le tableau 6.

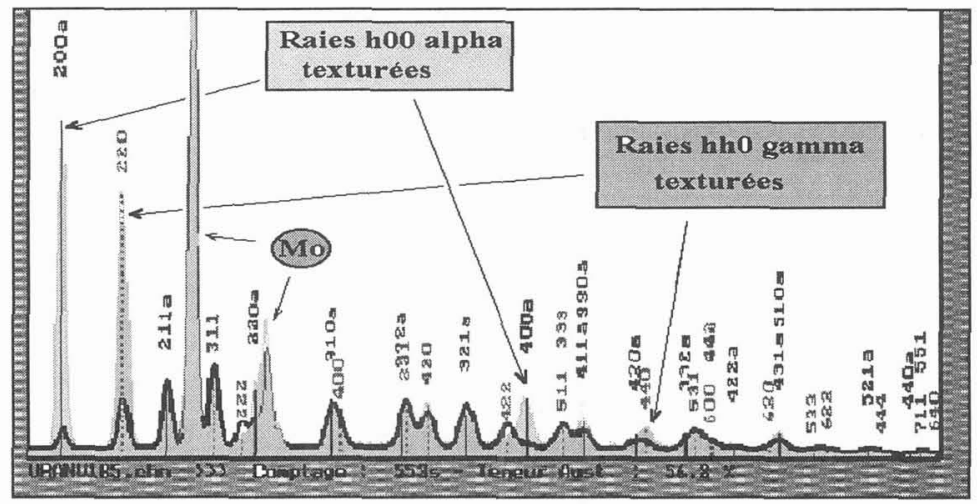

Figure $y$ : spectre ae lacier austeno-remrinque UKANUS $(\theta=18,2 \%)$.

Diffraction pattern of a URANUS designed austeno-ferritic steel. $\left(\theta=18,5^{\circ}\right)$.

Les mesures ont été faites à des angles variables, mais pour lesquels le nombre de raies est toujours élevé. La teneur calculée est pratiquement la même, quel que soit l'angle d'analyse, sauf à 14,5 où la raie de fluorescence du molybdène se superpose complètement à une raie intense du spectre, et perturbe ainsi l'analyse. 
Tableau 6 : Teneur en austénite dans l'acier austéno-ferritique texturé (voir figure 9), en fonction de l'angle $\theta$. Austenite content in an austeno-ferritic steel.with prefered orientation, (see fig 9), versus $\theta$.

\begin{tabular}{|c|c|c|c|c|c|c|c|c|c|c|c|c|}
\hline$\theta\left(^{\circ}\right)$ & 13 & 13,5 & 14 & $\mathbf{1 4 , 5}$ & 15 & 15,5 & 16 & 16,5 & 17 & 17,5 & 18 & 18,5 \\
\hline$\gamma_{\mathrm{R}} \%$ & 55,8 & 56,1 & 58,4 & $\mathbf{6 2 , 9}$ & 59,3 & 57,5 & 53,8 & 54,0 & 59,6 & 56,8 & 55,6 & 56,0 \\
\hline
\end{tabular}

Le bon résultat obtenu par la modélisation, quel que soit l'angle $\theta$ d'analyse, peut s'expliquer par le fait que seuls 2 couples de raies sont vraiment texturés. Même à $\theta=13^{\circ}$, le spectra contient déjà 21 raies, ce qui est apparemment suffisant pour que la modélisation corrige correctement l'effet de texture.

\section{CONCLUSION}

La modélisation du spectre de diffraction en dispersion d'énergie que nous avons réalisée est en parfait accord avec les spectres expérimentaux que nous avons obtenus sur des échantillons très divers. Elle conduit en outre à des résultats voisins de ce que l'on peut mesurer avec d'autres techniques. Elle peut donc être considérée comme un outil parfaitement au point. Grâce à cet outil, la technique de dosage de l'austénite par diffractométrie $\mathrm{X}$ en dispersion d'énergie se révèle un instrument d'analyse très puissant, dont le champ d'application a été considérablement étendu. Il est dorénavant possible de travailler à des angles élevés, où les raies se chevauchent complètement, ce qui permet de pondérer fortement les problèmes de textures. De plus, la plus grande partie des raies de carbures est éliminée, et celles qui subsistent dans le spectre ne viennent pas perturber le lissage. Le dosage de faibles teneurs en austénite ou en ferrite devient possible, même en présence d'une phase carbure importante. Le dosage est encore possible avec des spectres très mal définis, ce qui autorise des temps de comptage très courts ou des analyses sur de très petites zones. On peut estimer actuellement que la précision de l'analyse varie de $\pm 0,5 \%$ pour les faibles teneurs, à $\pm 2 \%$ pour de teneurs voisines de $50 \%$. Cette précision est équivalente, que l'on dose la ferrite ou l'austénite.

Grâce à la modélisation du spectre, la diffractométrie $\mathrm{X}$ en dispersion d'énergie devient un instrument d'avenir pour le dosage de l'austénite ou de la ferrite dans les aciers, aussi bien pour le laboratoire de recherche que pour le contrôle industriel.

\section{Références}

[1] Buras B. et al. Institute of Nuclear Research Varsovie (1968) Rep $n^{\circ}$ 894/II/PS

[2] Giessen B.C., Gordon G.E. Science 159 (1968) p.973

[3] Cole H. J. Apl. Cryst. 3 (1970) p.405

[4] Laine E. Kemian Teollisus 9 (1973) p.381

[5] Voskamp A.P. Advances in X-Ray Analysis 17 (1973) p.124

[6] Buras B. et al $A C T A$ Cryst. A31 (1975) p.327

[7] Guinier A., Théorie et technique de radiocristallographie (Dunod, 1964, 3 ième édition)

[8] Faninger G. et Hartmann H.T.M. 27 (1972)

[9] International Tables for X-Ray Cryst. (The Kynoch Press, Birmingham, 1968)

[10] Beu K.E. J. of Metals 194 (1952) p.1327

[11] Guiraldenq P. "Les possibilités actuelles de l'analyse aux rayons $\mathrm{X}$ de l'austénite résiduelle",VIe Journée des Aciers spéciaux (Toulouse, 8 juin 1967)

[12] Durnin J. et Ridal K.A., J.I.S.I. 206 part I(1968)

[13] Coulomb P., Les textures dans les métaux de réseau cubique (Dunod, 1972) 